\title{
Le financement des pensionnats de jeunes filles au Québec : le modèle de la congrégation des Soeurs de Sainte-Anne, 1850-1950
}

\section{Micheline Dumont et Lucie Champagne}

\section{Volume 53, 1986}

Le renouveau religieux à Montréal au XIX ${ }^{\mathrm{e}}$ siècle

URI : https://id.erudit.org/iderudit/1006971ar

DOI : https://doi.org/10.7202/1006971ar

\section{Aller au sommaire du numéro}

\section{Éditeur(s)}

Les Éditions Historia Ecclesiæ Catholicæ Canadensis Inc.

ISSN

0318-6172 (imprimé)

1927-7067 (numérique)

Découvrir la revue

Citer cet article

Dumont, M. \& Champagne, L. (1986). Le financement des pensionnats de jeunes filles au Québec : le modèle de la congrégation des Soeurs de Sainte-Anne, 1850-1950. Sessions d'étude - Société canadienne d'histoire de l'Église catholique, 53, 63-91. https://doi.org/10.7202/1006971ar

\section{Résumé de l'article}

Les recherches récentes en histoire de l'éducation ont établi le rôle central des congrégations religieuses enseignantes féminines au Québec pour le développement de l'instruction des filles. Toutefois, le financement de ce réseau reste une question encore inexplorée. Après avoir établi quelques distinctions structurelles entre les diverses congrégations, on avance l'hypothèse que les congrégations enseignantes fondées au Québec après 1840 constitue un modèle particulier. La congrégation des Soeurs de Sainte-Anne, fondée à Vaudreuil en 1850, est étudiée sur le plan du financement : développement institutionnel statut des institutions, titres de propriétés, revenus et dépenses. L'analyse montre qu'un modèle se dégage. Au XIX ${ }^{\mathrm{e}}$ siècle, la congrégation doit financer ses institutions par la formule de l'internat : elle n’ouvre que des pensionnats. Après 1900, les dépenses publiques pour les écoles la libèrent de cette obligation : jusqu'en 1950, elle n'ouvre que des écoles. Mais elle conserve ses anciens pensionnats et les transforme en institutions spécialisées (cours Lettres-Sciences, Écoles normales, Écoles ménagères, cours classique), financées majoritairement par les revenus de l'internat. Par ailleurs, le travail gratuit des religieuses dans les pensionnats assure l'économie de ce mode de financement. Il semble donc que l'instruction des filles a pu être développée au Québec en dehors des sources publiques de subventions.
Tous droits réservés @ Les Éditions Historia Ecclesiæ Catholicæ Canadensis Inc., 1986
Ce document est protégé par la loi sur le droit d'auteur. L’utilisation des services d'Érudit (y compris la reproduction) est assujettie à sa politique d'utilisation que vous pouvez consulter en ligne.

https://apropos.erudit.org/fr/usagers/politique-dutilisation/ 


\title{
Le financement des pensionnats de jeunes filles au Québec: le modèle de la congrégation des Sœurs de Sainte-Anne, 1850-1950
}

\author{
Micheline DuMONT \\ Lucie Champagne \\ Université de Sherbrooke
}

\section{RÉSUMÉ}

Les recherches récentes en histoire de l'éducation ont établi le rôle central des congrégations religieuses enseignantes féminines au Québec pour le développement de l'instruction des filles. Toutefois, le financement de ce réseau reste une question encore inexplorée. Après avoir établi quelques distinctions structurelles entre les diverses congrégations, on avance l'hypothèse que les congrégations enseignantes fondées au Québec après 1840 constitue un modèle particulier. La congrégation des Sceurs de Sainte-Anne, fondée à Vaudreuil en 1850, est étudiée sur le plan du financement: développement institutionnel, statut des institutions, titres de propriétés, revenus et dépenses. L'analyse montre qu'un modèle se dégage. $A u X I X^{e}$ siècle, la congrégation doit financer ses institutions par la formule de l'internat: elle n'ouvre que des pensionnats. Après 1900, les dépenses publiques pour les écoles la libèrent de cette obligation: jusqu'en 1950, elle n'ouvre que des écoles. Mais elle conserve ses anciens pensionnats et les transforme en institutions spécialisées (cours Lettres-Sciences, Écoles normales, Écoles ménagères, cours classique), financées majoritairement par les revenus de l'internat. Par ailleurs, le travail gratuit des religieuses dans les pensionnats assure l'économie de ce mode de financement. Il semble donc que l'instruction des filles a pu être développée au Québec en dehors des sources publiques de subventions.

«La conscience populaire a retenu que les communautés religieuses étaient riches, perception qui s'est probablement formée progressivement, au cours des années 1900-1930, à mesure que le territoire québécois se parsemait de couvents, d'hospices, et que s'élevait le montant des taxes 
foncières destinées à assurer les services municipaux dont ces immeubles jouissaient presque gratuitement ${ }^{1}$. Cette affirmation de Hamelin et Gagnon (que d'ailleurs il faudrait corriger: la multiplication des institutions commence bien avant 1900) est à confronter avec celle de la page précédente de leur livre de synthèse: "On ne sait à peu près rien sur les sources de revenus des communautés et sur leur budget de fonctionnement ${ }^{2}$. Seules de nouvelles recherches peuvent répondre aux interrogations causées par cette impression et cette ignorance.

Conscienies depuis lợgtêiñips quỉili fallait cxamincr cette question, les historiennes du Groupe de recherches en histoire de l'éducation des filles (GREF) viennent d'entreprendre un nouveau projet sur le financement de l'éducation des filles ${ }^{3}$. Ce faisant, elles contribueront à apporter des éléments de réponse aux questions que pose l'étude des ressources financières de l'Église au Québec.

\section{Des distinctions préalables à établir}

Au risque de paraitre affirmer des évidences, il faudrait, avant d'aborder notre sujet, énumérer un certain nombre de distinctions structurelles indispensables.

La première distinction est liée à l'histoire. Les congrégations fondées ou implantées en Nouvelle-France ayant obtenu des seigneuries ou des fiefs se trouvent à bénéficier, au départ, des titres fonciers qui les placent dans une catégorie à part. Qui plus est, la valeur des terrains qu'elles possèdent se trouve augmentée de la plus-value que leur confère leur localisation dans les centre-villes, au moment où démarre l'urbanisation. Qu'elles y restent ou qu'elles s'en départissent, elles y trouvent leur profit ${ }^{4}$. D'autre part, les congrégations féminines, bien tolérées par les autorités anglaises après 1760 , ont pu récupérer des sommes assez importantes sur leurs placements en France au début du $\mathrm{XIX}^{\mathrm{e}}$ siècle $^{5}$ et celles de Montréal ont longtemps

1 Jean Hamelin et Nicole Gagnon, Histoire du catholicisme québécois, Le XXe siècle, Tome I, 1898-1940, Montréal, Boréal Express, 1984, p. 269.

2 Ibid, p. 266.

3 Ce groupe de recherches a été constitué au printemps de 1981 et regroupe des professeures et étudiantes de l'Université du Québec à Montréal, de l'Université de Sherbrooke et de l'Université de Montréal. Il a reçu des subventions fédérales (CRSH) et provinciales (FCAC) de 1981 à 1983 et bénéficie en ce moment d'une subvention du FCAR (1985-1987).

4 Alyne Lebel, «Les propriétés foncières des Ursulines et le développement de Québec, 1854-1940", Cahiers de géographie du Québec, vol. 25, nº 64 (avril 1981), p. 119-132. Thèse de maîtrise du même titre, Université Laval.

5 Sur cette question particulière, aucune étude n'a, à ce jour, démêlé l'imbroglio des démarches de $\mathrm{M}$. Thavenet pour récupérer les placements en France des congrégations religieuses féminines entre 1820 et 1844 . Tous les chercheurs s'entendent pour associer $\mathrm{M}$. Thavenet à cette opération (Lemieux, Lahaise, Maurault, Paradis) mais les montants réels 
bénéficié de l'appui financier substantiel des Sulpiciens ${ }^{6}$. Quant aux congrégations masculines, on sait que la conquête britannique a eu sur elles des effets qui visaient à leur éventuelle disparition?.

Cette distinction n'élimine que huit congrégations mais on doit immédiatement ajouter que parmi les quelque 180 instituts qui apparaissent par la suite ${ }^{8}$, on ne peut traiter comme des éléments comparables les congrégations masculines dont les membres sont clercs et peuvent par conséquent exercer un ministère paroissial ou pastoral. En effet, le ministère paroissial ou tout simplement sacramentel (les intentions de messes) est source de revenus. Gabriel Dussault a estimé qu'en 1864, une cure pouvait rapporter $800 \$$ annuellement alors que le salaire de trois enseignants est estimé à $600 \$$. Cette affirmation se trouve confirmée par les recherches sur le revenu des cures dans le diocèse de Nicolet ${ }^{10}$.

Exclues du ministère de par leur sexe, les religieuses n'ont donc pas accès aux revenus que procurent les cures, les messes, les prédications, les responsabilités diocésaines ou pastorales. On objectera qu'elles s'assurent souvent du monopole des hosties, des cierges et des dentelles liturgiques mais on conviendra aussi que cet humble artisanat ne saurait être mis sur le même plan que les responsabilités cléricales.

On ne peut non plus considérer dans la même catégorie les congrégations fondées au Québec et celles venues de l'étranger. La dépendance d'une maison mère, en France par exemple, peut créer des différences considérables. Claude Langlois a établi des typologies fort variées, sur le plan de la richesse, pour les congrégations françaises au XIX siècle $^{11}$. Il n'est certes pas indifférent de rappeler que parmi les congrégations venues s'implanter au Québec figurent trois des plus riches congrégations françaises

récupérés sont difficiles à préciser. Voir Histoire de la Congrégation de Notre-Dame de Montréal, Montréal, 1941, vol. VI (1790-1822), p. 220-235, 239-245, 274-293; vol. VII (1822-1840), p. $18-19,34-43,120-138$.

6 Brian Young, In Its Corporate Capacity. The Seminary of Montreal as a Business Institution, 1816-1876, Montréal, McGill-Queen's, 1986.

7 Marcel Trudel, L'Église canadienne sous le régime militaire (1759-1764), Québec, Presses de l'Université Laval, 1956-1957 (2 tomes), passim.

8 Bernard Denault, «Sociographie générale des communautés religieuses au Québec (1837-1970)" dans Éléments pour une sociologie des communautés religieuses au Québec, Montréal et Sherbrooke, Presses de l'Université de Montréal, 1975. Voir Annexe II, p. 197-201.

9 Gabriel Dussault, Charisme et économie. Les cinq premières communautés masculines établies au Québec sous le régime anglais (1837-1870), Département de sociologie, Université Laval, 1981, p. 132.

10 Jean Roy, "Les revenus des cures du diocèse de Nicolet, 1885-1904", Société canadienne d'histoire de l'Église catholique, Sessions d'étude, 52 (1985), p. 51-67.

11 Claude Langlois, Le catholicisme au féminin. Les congrégations françaises à supérieure générale au XIX $X^{e}$ siècle, Paris, Cerf, 1984. Voir p. 343-395: "La fortune congréganiste». 
et l'une des moins riches ${ }^{12}$. D'autre part, les conditions financières d'implantation ne peuvent être toujours comparées aux conditions de fondation ${ }^{13}$.

Certes on peut faire l'hypothèse que les provincialats québécois ont acquis assez tôt leur autonomie financière, mais la seule étude disponible sur cette question concerne des congrégations masculines et les critères utilisés pour évaluer l'évolution des instituts s'organisent autour des responsabilités pastorales et peuvent difficilement s'appliquer aux congrégations féminines ${ }^{14}$. Il faudrait une étude semblable pour les congrégations féminines.

Enfin, l'œuvre principale de la congrégation doit être considérée dans l'analyse. D'abord, le statut canonique (moniales vs religieuses à vœux simples) impose une structure particulière: l'autonomie de chaque monastère et, dans le cas des contemplatives, des traditions monastiques dont on connaît mal les réalités financières. L'étude sur les dots des religieuses entreprise par Micheline D'Allaire s'arrête en $1800^{15}$. Par ailleurs, pour les congrégations à supérieures majeures, la situation est fort différente selon que la congrégation offre un service gratuit: hospitalisation, charité, protection ou un produit payant: éducation/instruction. Les premières devront très tôt avoir recours à l'intervention de l'État à cause des coûts énormes impliqués dans l'administration de leurs services. Par ailleurs, le cas des religieusesménagères, trompeusement nommées "auxiliaires du clergé», est plus complexe à analyser encore, leurs conditions d'engagement étant, à ce jour, virtuellement inconnues. Il en est de même pour les ordres missionnaires.

Toujours est-il que d'une distinction à l'autre, on peut poser l'hypothèse que les congrégations enseignantes fondées après 1840 au Québec représentent un modèle particulier dans l'ensemble des œuvres congréganistes. Aussi est-on justifiées d'en examiner le financement spécifique, d'autant plus qu'aucune congrégation masculine ne peut être introduite dans le corpus.

\section{Le financement de l'éducation au Québec}

Impossible d'aborder cette question sans rappeler un certain nombre de constantes qui caractérisent la période 1850-1950 en histoire de l'éducation. La lenteur de l'État à investir massivement dans l'éducation n'est plus à démontrer, pas plus que le rôle central joué par l'Église dans l'organisation,

12 Ibid., p. 384 et 390.

13 On ne peut ici qu'attendre le résultat des recherches de Guy Laperrière sur cette question. Voir dans $R H A F$, “'Persécution et exil', la venue au Québec des congrégations françaises, 1900-1914», vol. 36, no 3 (déc. 1982), p. 389-412.

14 Voir l'étude de Gabriel Dussault citée à la note 9.

15 Micheline D’Allaire, Les dots des religieuses au Canada français 1639-1800, Montréal, Hurtubise HMH, 1986. 
l'animation et la philosophie de l'enseignement à tous les niveaux, mais surtout de l'enseignement secondaire et supérieur. Au chapitre du financement toutefois, il importe peut-être de souligner quelques disparités. À tous les niveaux, dans tous les milieux, à toutes les étapes du processus, l'instruction des filles se développe avec des coûts minimes, quelle que soit l'institution qui finance. Quelques exemples suffiront. Au XIXe siècle, à Montréal, les Sulpiciens subventionnent bien différemment les écoles des sœurs et celles des frères ainsi qu'en fait foi le tableau I.

\section{TABLEAU I}

\section{Dépenses des Sulpiciens pour les écoles de Montréal}

\begin{tabular}{|c|c|c|c|c|}
\hline & \multicolumn{2}{|c|}{$\begin{array}{l}\text { Écoles de filles dirigées } \\
\text { par les Sœurs de la } \\
\text { Congrégation-de-Notre-Dame }\end{array}$} & \multicolumn{2}{|c|}{$\begin{array}{l}\text { Écoles de garçons dirigées } \\
\text { par les Frères des } \\
\text { écoles chrétiennes (1837) }\end{array}$} \\
\hline & $\begin{array}{l}\text { Dépenses } \\
\text { d'investissement } \\
\text { (construction, } \\
\text { agrandissement) }\end{array}$ & $\begin{array}{l}\text { Dépenses } \\
\text { annuelles } \\
\text { Moyennes de } \\
\text { fonctionnement }\end{array}$ & $\begin{array}{l}\text { Dépenses } \\
\text { d'investissement } \\
\text { (construction, } \\
\text { agrandissement) }\end{array}$ & $\begin{array}{l}\text { Dépenses } \\
\text { annuelles } \\
\text { Moyennes de } \\
\text { fonctionnement }\end{array}$ \\
\hline $1830-1839$ & & $510 \$ \times 10$ ans & - & - \\
\hline $1840-1849$ & $1192 \$ *$ & $3270 \$ \times 10$ ans & $?$ & $?$ \\
\hline $1850-1859$ & $\begin{array}{l}5480 \$ \\
2632 \$\end{array}$ & $?$ & $7968 \$$ & $7998 \$ \times 10$ ans \\
\hline $1860-1869$ & $\begin{array}{r}4250 \$ \\
22272 \$ \\
1562 \$\end{array}$ & $?$ & $\begin{array}{l}25965 \$ \\
19013 \$\end{array}$ & $?$ \\
\hline $1870-1879$ & $2530 \$$ & $7930 \$ \times 10$ ans & $\begin{array}{l}24281 \$ \\
29271 \$\end{array}$ & $15847 \$ \times 10$ ans \\
\hline $\begin{array}{l}\text { Total } \\
\text { approx. }\end{array}$ & $38726 \$$ & & $106498 \$$ & \\
\hline
\end{tabular}

SOURCE: Brian Young, In Its Corporate Capacity. The Seminary of Montreal as a Business Institution, 1816-1876, Appendice 17.

* Reconstruction avec les assurances après un incendie. 
À la même époque, la C.E.C.M. établit la même distinction entre les écoles laïques de filles et de garçons. Marta Danylewycz a démontré que les commissaires dépensent en 1881 plus de $10 \$$ par garçon à l'école chaque année alors que ces dépenses sont inférieures à $2,50 \$$ par fille à l'école ${ }^{16}$. Au début du XXe siècle, la somme des subventions accordées par le D.I.P. à 50 pensionnats féminins équivaut souvent à celle que reçoit un seul des collèges classiques (uniquement masculins, faut-il le rappeler...) ${ }^{17}$. En 1945, 39 écoles normales de garçons se partagent $150833 \${ }^{18}$. Par ailleurs, on sait que les collèges classiques féminins n'ont pas bénéficié de subventions avant le début des années $1960^{19}$. Et que dire des salaires des institutrices durant plus d'un siècle à travers tout le Québec? ${ }^{20}$

Le développement de l'instruction des filles au Québec a été assuré par les congrégations religieuses féminines. Les recherches du GREF, qui viennent d'être publiées dans notre livre Les couventines ${ }^{21}$, ont abondamment documenté le rôle central des congrégations pour allonger les programmes d'études; concevoir et diversifier ces programmes; mettre sur pied et faire fonctionner les structures administratives et pédagogiques; encadrer et instruire les générations successives d'étudiantes; parsemer le territoire québécois de centaines d'institutions. Au delà du niveau primaire, jusqu'en 1954, la scolarisation plus avancée des filles est assurée presque exclusivement par les religieuses 22 .

La question principale reste toutefois sans réponse: comment s'est financé ce réseau complexe et multiforme? Nous rejoignons ici notre interrogation initiale et c'est par l'étude d'une congrégation particulière fondée au Québec après 1840 que nous voulons tenter de répondre à la question. Les Sœurs de Sainte-Anne ont généreusement accepté de nous

16 Marta Danylewycz, «Sexes et classes sociales dans l'enseignement: le cas de Montréal à la fin du 19e siècle", dans Nadia Fahmy-Eid et Micheline Dumont, Mâttresses de maison, maitresses d'école. Femmes, famille et éducation dans l'histoire du Québec, Montréal, Boréal Express, 1983, p. 103. Ruby Heap vient de faire la même constatation: voir «Les femmes laïques au service de l'enseignement primaire public catholique à Montréal: les écoles des dames et demoiselles" dans Canadian Women Studies/Les cahiers de la femme, vol. 7, no 3 (automne 1986), p. 55-60.

17 Rapport du Surintendant de l'Instruction publique, 1906-1907, p. 312-349.

18 Ibid., 1945-1946, p. IX.

19 La signification et le besoin de l'enseignement classique pour jeunes filles dans le Québec. Mémoire des collèges classiques de jeunes filles du Québec à la Commission royale d'enquête sur les problèmes constitutionnels, Montréal, Fides, 1954, p. 12.

20 Maryse Thivierge, «La syndicalisation des institutrices catholiques, 1900-1959" dans Maitresses de maison, maitresses d'école, p. 171-190.

21 Micheline Dumont et Nadia Fahmy-Eid, Les couventines. L'éducation des filles au Québec dans les congrégations religieuses enseignantes 1840-1960, Montréal, Boréal, 1986, $318 \mathrm{p}$.

22 Micheline Dumont, "L'instruction des filles avant 1960" dans Interface, vol. 7, n 3 (mai-juin 1986), p. 22-29. 
ouvrir leurs archives, mais à travers elles c'est treize congrégations distinctes que nous pouvons peut-être appréhender ${ }^{23}$. Ajoutons tout de suite que ces treize congrégations sont très représentatives puisqu'elles comprenaient, en 1969,15015 religieuses, soit la moitié de toutes les religieuses enseignantes et le tiers de l'ensemble des religieuses québécoises. La congrégation des Sœurs de Sainte-Anne, fondée à Vaudreuil en 1850, avec ses 2000 sujets en 1969 , se présente donc comme un microcosme intéressant dont le modèle de développement pourrait être appliqué, sinon généralisé, à l'ensemble des pensionnats québécois. De toutes manières, elle représente une des plus importantes congrégations par le nombre de ses maisons, le nombre des religieuses enseignantes et par l'ensemble de ses initiatives pédagogiques ${ }^{24}$. Nous avons arrêté la recherche à 1950 , car c'est après cette date que des modifications substantielles interviennent au chapitre des investissements publics en éducation ${ }^{25}$.

\section{Le développement institutionnel de la congrégation}

À sa fondation en 1850, la Congrégation des Sœurs de Sainte-Anne sétablit à Vaudreuil, en région périphérique de Montréal. Dès 1858, elle est amenée à fonder une première mission à l'extérieur du Québec, soit à Victoria dans le territoire qui deviendra la Colombie-Britannique ${ }^{26}$. Par la suite, elle ouvre de nouveaux établissements dans l'Ouest canadien, aux États-Unis, ainsi qu'au Japon et en Haiti au XXe siècle. Les fondations au

23 Il s'agit de l'ensemble des congrégations féminines enseignantes fondées au Québec après 1840. En voici la liste:

1843 Sœurs des Saints Noms de Jésus et Marie (4011)

1849 Sceurs de la Charité de Québec (1846)

1850 Servantes du Cœur Immaculé de Marie (1426)

1850 Scurs de Sainte-Anne (2021)

1853 Sœurs de l'Assomption-de-la-Sainte-Vierge (1762)

1874 Sours de Notre-Dame-du-Saint-Rosaire (935)

1877 Sours de Saint-Joseph de Saint-Hyacinthe (698)

1883 Sours de Notre-Dame de Sainte-Marthe (197)

1891 Petites Franciscaines de Marie (612)

1892 Sœurs de Notre-Dame du Perpétuel Secours (810)

1894 Sœurs de Notre-Dame-du-Bon-Conseil de Chicoutimi (515)

1921 Sœurs de Notre-Dame Auxiliatrice (160)

1950 Sœurs des Saints Apôtres (22)

Le chiffre entre parenthèses indique le nombre total de sujets dans toute la congrégation en 1969. Source: Bernard Denault, op. cit.

24 Augustine Prévost, L'éducation hier et aujourd'hui, Montréal, Éditions du Méridien, 1986. Cet ouvrage aborde l'évolution de l'enseignement chez les Sœurs de Sainte-Anne.

25 Linteau, Durocher, Robert, Ricard, Histoire du Québec contemporain, Tome II: Le Québec depuis 1930, Montréal, Boréal, 1986, p. 316-317.

26 Liste des établissements de l'Institut des Sceurs de Sainte-Anne, 1850-1976, Lachine, Archives des Sœurs de Sainte-Anne (ASSA). 
Québec constituent $56 \%$ de toutes les maisons d'éducation ${ }^{27}$ ouvertes entre 1850 et 1950; c'est exclusivement de ces établissements qu'il sera question ici. Le tableau II en présente la liste: son examen permet quelques observations.

La première a pour objet la stabilité des fondations. La majorité de ces maisons fondées au Québec sont toujours ouvertes en 1950, à l'exception de quatre, soit deux nensionnats et deux écoles ${ }^{28}$. La fermeture est causée soit par un manque de revenus, soit par une clientèle étudiante insuffisante ${ }^{29}$. Sur un total de soixante-quatorze fondations, seulement quatre ont dû fermer leurs portes, et ce, pour des raisons incontrôlables par la congrégation. Les Sœurs de Sainte-Anne ont maintenu leur objectif d'éducation pour les jeunes filles; leurs institutions demeurent et la congrégation abandonne rapidement celles qui n'ont pas de chance de subsister. Les fondations se caractérisent donc par la stabilité et le réalisme.

La deuxième observation concerne la périodisation des fondations. Le rythme des maisons ouvertes au Québec est régulier de 1850 à 1950, excepté pour deux périodes. La première se distingue facilement: il y a un net ralentissement après la fondation du pensionnat de Ste-Angèle à SteCunégonde en 1888, ralentissement qui se poursuit jusqu'à la nouvelle vague de fondations débutant avec l'ouverture de l'Académie Savaria à Lachine en 1906. À l'intérieur de ces dix-huit années, il n'y a que deux fondations: le pensionnat de L'Ange-Gardien à St-Henri en 1898 et l'Académie MarieAnne à St-Jean-de-la-Croix en 1902.

Après une première vague de fondations de 1850 à 1888 , période considérée comme l'expansion originelle, cette rupture dans le rythme des fondations n'en est pas vraiment une pour la congrégation. Premièrement, ce fut pour l'institution des Sœurs de Ste-Anne une période de reconstruction et d'agrandissement des pensionnats déjà établis au Québec ${ }^{30}$. Deuxièmement, la congrégation ouvre dix-sept maisons d'éducation à l'extérieur du Québec durant ce laps de temps, principalement aux États-Unis et dans l'Ouest canadien ${ }^{31}$.

27 Ibid., p. 1-16.

28 Il s'agit du couvent d'Hemmingford (1870-1884), du couvent de St-Venant-de-Hereford (1877-1884), de l'École Ste-Marie Salomé dans le comté de Montcalm (1919-1922) et de l'École St-Nazaire de Ville Lasalle (1920-1924).

$29 \mathrm{Sr}$ Marie-Jean-de-Pathmos, Les Sœeurs de Ste-Anne, un siècle d'histoire, Tome I, 1850-1900, Lachine, S.S.A., 1950, p. 238 et 256 et Rapports du Surintendant de l'Instruction publique, Québec, Département de l'instruction publique, 1919-1922.

$30 \mathrm{Sr}$ Marie-Jean-de-Pathmos, op. cit., et Abbé Elie-J. Auclair, Histoire des Soeurs de Ste-Anne. Les premiers cinquante ans, 1850-1900, Montréal, 1922.

31 Archives des Sœurs de Sainte-Anne (désormais ASSA), Liste des établissements de l'Institut des Sceurs de Ste-Anne, p. 1-9. 


\section{TABLEAU II}

Liste des établissements d'éducation par année de fơndation, Sœurs de Sainte-Anne (S.S.A.), Province de Québec, 1850-1950

\begin{tabular}{|c|c|c|c|}
\hline $\begin{array}{c}\text { Année } \\
\text { de } \\
\text { fondation }\end{array}$ & NOM DES ÉTABLISSEMENTS & $\begin{array}{c}\text { Année } \\
\text { de } \\
\text { fondation }\end{array}$ & NOM DES ÉTABLISSEMENTS \\
\hline 1850 & Ct Vaudreuil & 1900 & \\
\hline 1851 & Ct Ste-Geneviève & 1901 & \\
\hline 1852 & & 1902 & Ac St-Jean-de-la-Croix \\
\hline 1853 & Ct St-Jacques & 1903 & \\
\hline 1854 & & 1904 & \\
\hline 1855 & Ct St-Ambroise & 1905 & \\
\hline 1856 & & 1906 & Ac Savaria, Ac Esther-Blondin \\
\hline 1857 & Ct St-Cyprien & 1907 & Ac St-Michel, Ac Martin \\
\hline 1858 & & 1908 & Une Académie, deux Écoles \\
\hline 1859 & Ct Rigaud & 1909 & Ac St-Georges \\
\hline 1860 & & 1910 & \\
\hline 1861 & Ct Lachine & 1911 & Éc N-D-des-Victoires, Éc St-Victor \\
\hline 1862 & & 1912 & Deux Écoles \\
\hline 1863 & & 1913 & Éc Ste-Clotilde \\
\hline 1864 & Ct St-Jérôme & 1914 & \\
\hline 1865 & Ct Rawdon, Ct St-Michel & 1915 & Éc Caughnawaga \\
\hline 1866 & Ct St-Gabriel de Brandon & 1916 & Éc Dorion \\
\hline 1867 & Ct St-Polycarpe & 1917 & Éc St-Cuthbert, Éc St-Esprit \\
\hline 1868 & & 1918 & Une Académie, quatre Écoles \\
\hline 1869 & & 1919 & Éc Ste-Marie Salomé \\
\hline 1870 & Ct Hemmingford, Ct St-Henri & 1920 & Ac St-Thomas-d'Aquin, Éc St-Nazaire \\
\hline 1871 & Ct St-Rémi & 1921 & Éc Ste-Herménégilde \\
\hline 1872 & & 1922 & Éc St-Constant, Éc Val-David \\
\hline 1873 & & 1923 & Trois Écoles \\
\hline 1874 & Ct St-Cuthbert & 1924 & Éc St-Joseph, Éc St-Jean-Baptiste \\
\hline 1875 & & 1925 & \\
\hline 1876 & Ct St-Esprit & 1926 & \\
\hline 1877 & Ct St-Venant-de-Hereford & 1927 & \\
\hline 1878 & & 1928 & Éc Ménagère Régionale St-Jacques \\
\hline 1879 & Ct Ste-Cunégonde & 1929 & Éc St-Lquis \\
\hline 1880 & & 1930 & Éc Holyl Family \\
\hline 1881 & & 1931 & Éc Daignault. \\
\hline 1882 & & 1932 & Cours classique Marie-Anne \\
\hline 1883 & Ct Ste-Anne-des-Plaines & 1933 & \\
\hline 1884 & & 1934 & Éc Saraguay \\
\hline 1885 & Ct St-Félix-de-Valois & 1935 & \\
\hline 1886 & & 1936 & \\
\hline 1887 & Ct St-Norbert & 1937 & \\
\hline 1888 & Ct Ste-Angèle & 1938 & Éc Ste-Bernadette \\
\hline 1889 & & 1939 & \\
\hline
\end{tabular}


TABLEAU II (suite)

Liste des établissements d'éducation par année de fondation, Sœurs de Sainte-Anne (S.S.A.), Province de Québec, 1850-1950

\begin{tabular}{|c|c|c|l|}
\hline $\begin{array}{c}\text { Année } \\
\text { de } \\
\text { fondation }\end{array}$ & NOM DES ÉTABLISSEMENTS & $\begin{array}{c}\text { Année } \\
\text { de } \\
\text { fondation }\end{array}$ & NOM DES ÉTABLISSEMENTS \\
\hline & & 1940 & Éc Supérieure de Musique à Lachine \\
1890 & & 1941 & \\
1891 & & 1942 & Éc Supérieure St-Jean-de-la-Croix \\
1892 & & 1943 & Éc St-Régis \\
1893 & & 1944 & \\
1894 & & 1945 & \\
1895 & & 1946 & \\
1896 & & 1947 & Ac Ste-Anne \\
1897 & Ct L'Ange-Gardien & 1948 & Éc secondaire St-Thomas, Éc norm. Rigaud \\
1898 & & 1949 & Éc Thérien, Ec Dupuis, Ec Ste-Rose \\
1899 & &
\end{tabular}

Source: ASSA, Liste des établissements des S.S.A., révisée en 1968.

Abréviations: Ct: couvent Ac: académie Éc: école

Les noms en italique sont ceux des établissements qui ont fermé leurs portes avant 1950.

La deuxième période où l'on observe un ralentissement dans le rythme des fondations est plus évidente si nous observons le phénomène en regroupant les maisons fondées par tranche de dix ans (voir graphique I). Sur le graphique, il y a deux baisses distinctes: soit la décennie 1890-1899 avec un seul établissement et représentant la première rupture, et celle de la décennie 1930-1939. Ce phénomène s'explique par la crise économique qui, au début des années 1930, a ralenti non seulement l'expansion de la congrégation, mais celle du pays tout entier. Toutefois, cette période correspond à la plus grande progression dans le nombre de religieuses professes $^{32}$.

La périodisation par rapport au développement des maisons d'éducation de la congrégation des Sœurs de Ste-Anne au Québec est donc la suivante:

Années

1850-1888

1889-1905

1906-1924

1925-1946

1947-1964

\section{Période}

expansion originelle consolidation

nouvelle expansion

période de crise et de guerre expansion récente
Nombre de fondations

$$
\begin{array}{r}
23 \\
2 \\
33 \\
10 \\
49
\end{array}
$$

Une troisième observation sur les établissements d'éducation des Sœurs de Ste-Anne au Québec démontre qu'ils sont localisés en

32 ASSA, Matricule des S.S.A., Lachine, 1968. 


\section{GRAPHIQUE}

Fondations des établissements d'éducation par décennie, S.S.A., Province de Québec, 1850-1950

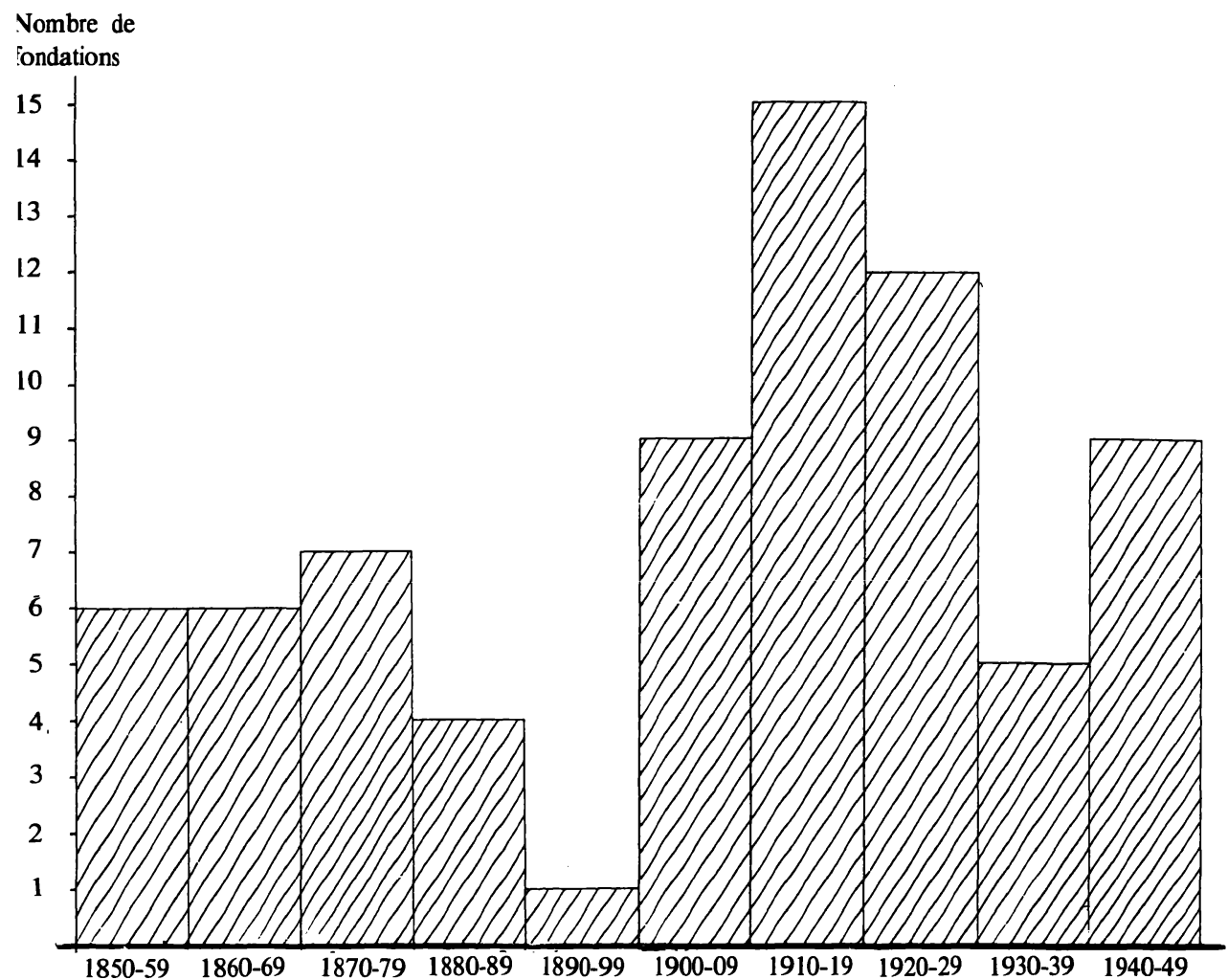

?ondations 
majorité dans une seule région de la province: Montréal et ses environs (voir carte). Sur les soixante-quatorze maisons fondées entre 1850 et 1950, plus de la moitié, soit quarante, se situent dans le diocèse de Montréal; d'autres diocèses se partagent le reste de la façon suivante: douze maisons dans le diocèse de Joliette, dix dans le diocèse de St-Jean, neuf dans le diocèse de Valleyfield, tous issus du diocèse de Montréal, et les trois dernières se répartissent dans les diocèses de Mont-Laurier, d'Amos et de Sherbrooke.

Près de $43 \%$ des institutions ont été fondées sur l'île de Montréal, en milieu urbain. Les autres établissements, soit $57 \%$, sont situés en milieu rural et rencontrent les objectifs premiers de la congrégation, fondée pour donner l'enseignement aux jeunes filles dans les milieux ruraux ${ }^{33}$. À l'exception des deux institutions situées dans les diocèses d'Amos et de Sherbrooke, l'ensemble des fondations dans la province de Québec est situé à l'intérieur d'un rayon de $70 \mathrm{~km}$ autour de l'île de Montréal. De ce nombre, seulement trois institutions se trouvent au-delà de $60 \mathrm{~km}$, et le train accède facilement à deux de ces maisons, soit Val-David et St-Gabriel-de-Brandon. L'examen de la carte fait voir également qu'il n'y a aucune institution des Sœurs de Sainte-Anne à l'Est de Montréal. Cela est dû à l'implantation ou à la fondation de plusieurs autres congrégations féminines enseignantes dans cette région, telles que les Sœurs de la Présentation de Marie, les Sœurs des Saints-Noms de Jésus et de Marie et les Sœurs de St-Joseph de St-Hyacinthe.

En dernier lieu, l'examen de la liste des établissements d'enseignement des Sœurs de Sainte-Anne au Québec démontre que la congrégation a fondé différents types de maisons autres que le pensionnat et l'école paroissiale, tels des écoles normales, un collège classique et des écoles ménagères. Par exemple, elle fonde une école normale à Saint-Jérôme en 1923 et une deuxième à Rigaud en 1948. Ces deux institutions font partie de la grande vague de fondations des écoles normales débutée en 1907 par celle des Ursulines de Trois-Rivières ${ }^{34}$. De la même façon, les Sœurs de Sainte-Anne ouvrent les portes aux jeunes filles qui veulent suivre le cours classique à St-Henri en 1932, institution qui sera par la suite transférée à Lachine en 1941. Cette fondation inaugure l'ère de multiplication des collèges classiques féminins dans la région de Montréal ${ }^{35}$. Elles ouvrent également une école ménagère régionale à St-Jacques en 1928, qui deviendra une école supérieure d'enseignement ménager en 1949. Plusieurs de leurs pensionnats offrent aussi quelques cours faisant partie du programme de l'enseignement ménager.

33 Sr Marie-Jean-de-Pathmos, op. cit., p. 65. p. 65 .

34 Jeannette Létourneau, Les écoles normales de filles au Québec, Montréal, Fides, 1981,

35 Claude Galarneau, Les collèges classiques au Canada français, Montréal, Fides, 1978, p. 56. 
Établissements d'éducation des Sours de Sainte-Anne dans la région montréalaise en 1950

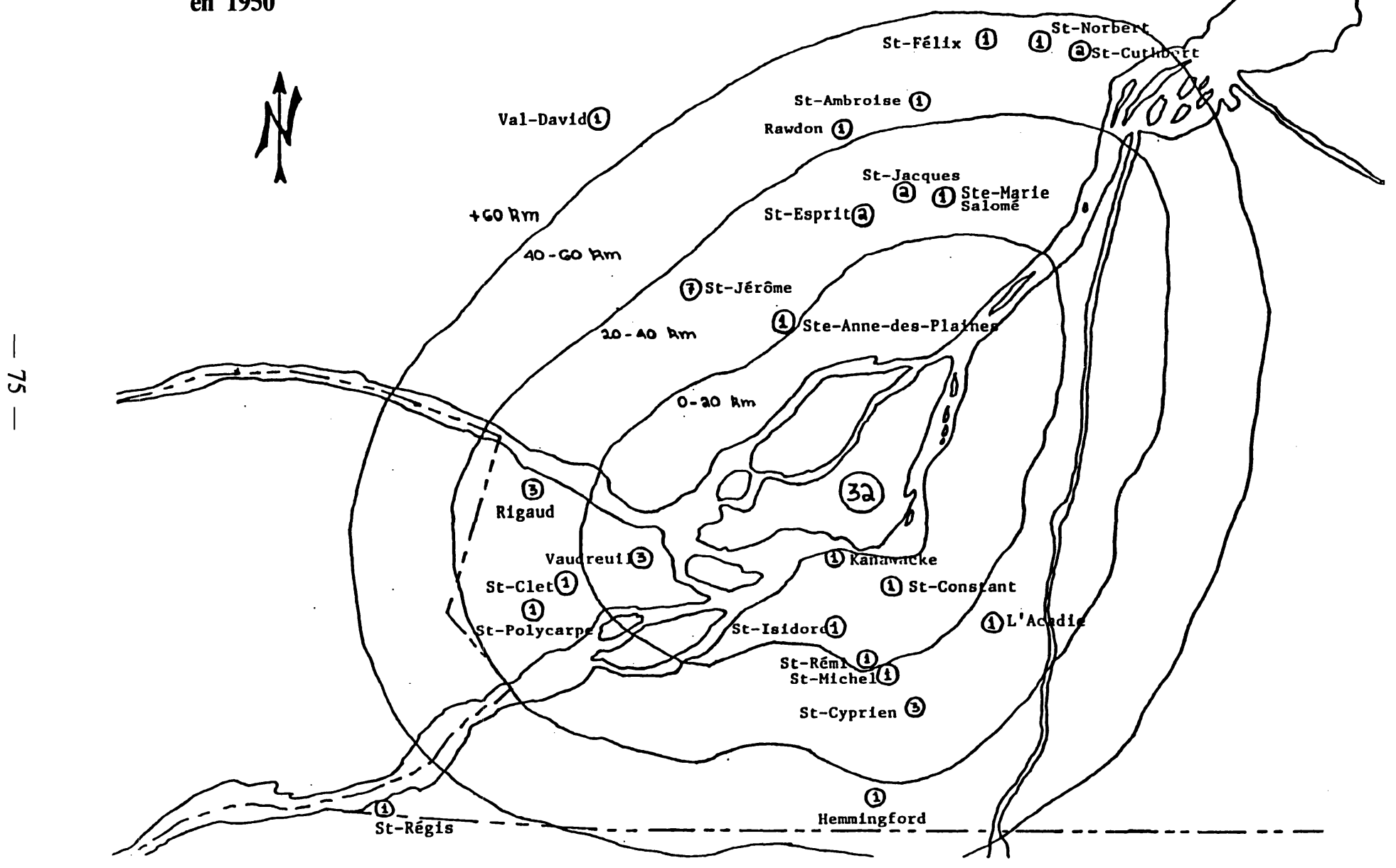


Avec toutes ces fondations, les Sœurs de Sainte-Anne suivent un développement semblable à celui de plusieurs congrégations féminines enseignantes au Québec ${ }^{36}$; elles s'adaptent à la conjoncture éducative de l'époque. Toutes les institutions qui allongent la scolarité des programmes destinés aux filles ont été fondées par des congrégations. Ces religieuses ont donné la chance aux jeunes filles d'accéder à des niveaux d'étude qui leur étaient interdits auparavant, et qui leur auraient été inaccessibles sans le travail et l'investissement de ces congrégations religieuses. Il ne faut pas oublier toutefois que seule une minorité de jeunes filles ont pu atteindre ces dégrés supérieurs.

\section{Le statut des institutions des Soeurs de Sainte-Anne}

Au chapitre des sources de financement, tous les établissements de la congrégation des Sœurs de Sainte-Anne n'ont pas le même statut. On peut distinguer les institutions "indépendantes", les institutions "sous contrôle» et les institutions «indépendantes et sous contrôle ${ }^{37}$.

Les premières, soit les institutions «indépendantes", sont les établissements où les religieuses assument la direction, l'enseignement et l'administration de l'institution. Ces maisons reçoivent des subventions provenant du fonds de l'éducation supérieure, si minimes soient-elles ${ }^{38}$. Elles accueillent des pensionnaires, des demi et des quart de pension, et ce sont les parents qui défraient les coûts de la pension et de l'enseignement. Elles sont considérées comme des écoles privées et atteignent ou dépassent habituellement le diplôme académique, plus haut degré auquel les filles pouvaient théoriquement accéder jusqu'en 1923, à l'école publique.

Dans les institutions «sous contrôle", les religieuses assument la direction et l'enseignement, mais les écoles sont administrées par les commissaires; les religieuses enseignantes y reçoivent un salaire. Les coûts de l'enseignement sont défrayés par la commission scolaire, et souvent, les parents participent aux frais en payant une rétribution mensuelle. Ces établissements sont des écoles publiques; ils accueillent les enfants de situation plus modeste et le niveau d'étude plafonne généralement au cours élémentaire ou au cours modèle dans certaines villes.

Les institutions «indépendantes et sous contrôle" sont des établissements où l'on retrouve deux types de clientèle dans la même maison. D'un côté, les classes privées payantes, donc des classes à niveau plus avancé, où le matériel didactique, l'ameublement et les services sont de meilleure qualité;

36 Micheline Dumont et Marie-Paule Malouin, “Évolution et rôle des congrégations religieuses enseignantes féminines au Québec, 1840-1960”, S.C.H.E.C., Sessions d'étude, 50 (1983), vol. I, p. 202-205.

37 Cette terminologie est celle des Rapports $d u$ Surintendant de l'Instruction publique.

38 Micheline Dumont, "L'instruction des filles avant 1960" dans Interface, vol. 7, n 3, mai-juin 1986, p. 24-25. 
et de l'autre côté les classes publiques, donc les classes à niveau moins avancé et où le ratio élèves-professeur est souvent plus élevé qu'à l'école privée.

$\mathrm{Au}$ moment de compter le nombre d'institutions des Sœurs de SainteAnne pour chacune des trois catégories, des problèmes méthodologiques ont surgi. Trois sources distinctes permettaient d'effectuer ces calculs, mais les résultats différaient. Le choix du document retenu s'est arrêté sur le Registre des décrets de la Cour de Rome et des Rapports triennaux adressés à la Sacrée Congrégation des Clercs et Réguliers ${ }^{39}$ pour deux raisons: premièrement, parce que la source provient des archives de la communauté, et deuxièmement parce qu'elle est la plus constante.

Parmi les maisons d'éducation des Sœurs de Sainte-Anne au Québec, ce sont les institutions indépendantes et sous contrôle qui sont majoritaires à la fin du XIX $\mathrm{X}^{\mathrm{e}}$ siècle et au début du XXe siècle: elles comptent pour $74 \%$ des maisons. Mais à cause des nombreuses écoles ouvertes par les religieuses au $\mathrm{XX}^{\mathrm{e}}$ siècle, ces institutions passeront sous les $43 \%$ du total des maisons d'éducation au début des années 1930, tandis que les écoles sous contrôle s'approcheront du 60\%. Notons que les Sœurs de Sainte-Anne ne fondent aucune institution indépendante après cette date, si ce n'est le Collège MarieAnne, nom de leur collège classique.

Ce modèle d'institution, un pensionnat jumelé à une école paroissiale, signifie qu'à l'intérieur de la même institution se côtoient deux groupes différents, deux classes sociales. Avec le dédoublement du réseau scolaire, les religieuses auront permis à une minorité de jeunes filles d'accéder à des niveaux d'étude de plus en plus élaborés. En allongeant leur formation scolaire, les religieuses enseignantes ont par le fait même transformé le rôle des filles dans la société et aidé à changer les valeurs traditionnelles qui ont longtemps conditionné ces jeunes filles au rôle d'épouse, de mère et de maîtresse de maison. Elles ont ainsi favorisé l'émancipation des femmes, mais ce faisant, elles se trouvaient à maintenir plus longtemps la clientèle au pensionnat. Ce n'est qu'au milieu du XXe siècle qu'un nombre plus élevé de jeunes filles pourra accéder à ces niveaux supérieurs avec l'instauration d'un système public d'enseignement ${ }^{40}$. On peut d'ores et déjà s'interroger sur la cause de la longue (1850-1930) prépondérance numérique des institutions «indépendantes et sous contrôle». Nous tenterons d'y répondre en examinant deux questions: celle de la propriété foncière et celle du budget de fonctionnement.

39 ASSA, Registre des décrets de la Cour de Rome et des rapports triennaux adressés à la Sacrée Congrégation des Clercs et Réguliers, 1864 à 1946. Les autres sources sont les Rapports du Surintendant de l'Instruction publique (désormais R.S.I.P.) et le Canada ecclésiastique.

40 Micheline Dumont et Nadia Fahmy-Eid, Les couventines, p. 189-225. 


\section{La propriété des institutions des Sœurs de Sainte-Anne}

L'examen de la question de la propriété foncière des institutions chez les Sœurs de Sainte-Anne pose un nouveau problème méthodologique: dans tous les documents utilisés jusqu'à maintenant, il n'est presque jamais fait mention de la propriété des établissements. Une seule source indique la propriété de chacune des maisons d'enseignement: la Liste des établissements d'éducation, propriété et usufruit ${ }^{41}$. Malheureusement, cette liste n'existe que pour l'année 1902.

Le tableau III reproduit la liste de ce document. La première catégorie comprend les maisons appartenant à la congrégation, c'est-à-dire les établissements dont l'Institut a fait l'achat des terrains et des bâtisses. La deuxième catégorie englobe les institutions dont la congrégation n'a que l'usufruit; même si elles y habitent, les terrains et les bâtisses n'appartiennent pas aux religieuses, mais à la municipalité scolaire ou à la fabrique. Ces maisons sont cédées aux religieuses tant et aussi longtemps qu'elles enseigneront aux enfants de la paroisse. Le jour où elles cesseront cette activité, les religieuses devront remettre les terrains et les bâtisses au propriétaire. Les revenus de ces maisons sont toutefois administrés par les religieuses, et les dettes contractées sont remboursées par la congrégation. Finalement, les établissements où les religieuses n'habitent pas constituent la troisième catégorie. Ces maisons appartiennent soit à la municipalité scolaire, soit à la fabrique, et les religieuses n'y font que l'enseignement.

La majorité des établissements de la première et de la deuxième catégorie comprennent un pensionnat et un externat. Les deux exceptions, Ste-Cunégonde et St-Henri, ont également une école paroissiale jumelée à leur pensionnat; mais ces écoles figurent justement dans la troisième catégorie, car les bâtisses de l'externat n'appartiennent pas à la congrégation.

L'examen des fondations des Sœurs de Sainte-Anne démontre que leur dernier pensionnat est instauré en 1898. Après cette date, elles ne fondent que des écoles ou bien agrandissent ou transforment les pensionnats existants. Manifestement, au XIX ${ }^{\mathrm{e}}$ siècle, les religieuses semblent contraintes d'ouvrir un pensionnat à chaque établissement: les pensions des élèves constituent une source de financement pour l'institution. Au XXe siècle, cette obligation disparaît-elle? Peut-on expliquer la rupture dans le type de fondations?

On en trouve vraisemblablement l'explication dans l'évolution des dépenses publiques pour l'éducation. Au seul chapitre de la construction des écoles, les dépenses publiques sont multipliées par trois entre 1904 et $1909^{42}$.

41 ASSA, Liste des établissements d'éducation, propriété et usufruit, 1902.

42 R.S.I.P., 1904-1905 et 1909-1910. 


\section{TABLEAU III}

Liste des établissements d'éducation, propriété et usufruit, S.S.A., Province de Québec, 1902

\section{Maisons appartenant à l'institut}

\begin{tabular}{|c|c|c|}
\hline Année de fondation & Nom de l'établissement & $\begin{array}{c}\text { Nature de } \\
\text { l'établissement }\end{array}$ \\
\hline 1853 & St-Jacques & pens. - ext. \\
1857 & St-Cyprien & pens. - ext. \\
1859 & Rigaud & pens. - ext. \\
1861 & Lachine & pens. - ext. \\
1865 & Rawdon & pens. - ext. \\
1874 & St-Cuthbert & pens. - ext. \\
1888 & Ste-Cunégonde & pens. \\
1898 & St-Henri & pens. \\
\hline
\end{tabular}

\section{Maisons dont l'institut n'a que l'usufruit}

\begin{tabular}{|c|c|c|}
\hline Année de fondation & Nom de l'établissement & $\begin{array}{c}\text { Nature de } \\
\text { 'établissement }\end{array}$ \\
\hline 1850 & Vaudreuil & pens. - ext. \\
1851 & Ste-Geneviève & pens. - ext. \\
1855 & St-Ambroise & pens. - ext. \\
1864 & St-Jérôme & pens. - ext. \\
1865 & St-Michel & pens. - ext. \\
1866 & St-Gabriel-de-Brandon & pens. - ext. \\
1867 & St-Polycarpe & pens. - ext. \\
1871 & St-Rémi & pens. - ext. \\
1876 & St-Esprit & pens. - ext. \\
1883 & Ste-Anne-des-Plaines & pens. - ext. \\
1885 & St-Félix-de-Valois & pens. - ext. \\
1887 & St-Norbert & pens. - ext. \\
\hline
\end{tabular}

3. Maisons appartenant aux municipalités scolaires ou aux églises paroissiales

\begin{tabular}{|c|c|c|}
\hline Année de fondation & Nom de l'établissement & $\begin{array}{c}\text { Nature de } \\
\text { l'établissement }\end{array}$ \\
\hline 1870 & St-Henri & ext. \\
1879 & Ste-Cunégonde & ext. \\
1902 & St-Jean-de-la-Croix & ext. \\
1902 & Ste-Élizabeth & ext. \\
\hline
\end{tabular}

Source: Liste des établissements envoyée à Rome en 1902.

pens.: pensionnat

ext.: externat 
De leur côté, les contributions générales scolaires suivent la progression suivante (voir tableau IV): les taxes passent de $25,9 \%$ à $62 \%$ en l'espace de 60 ans, les rétributions mensuelles tombent de vint-huit points de pourcentage en l'espace de vingt ans, s'approchant ainsi du 5\% au début du XXe siècle, et les contributions des institutions indépendantes demeurent stables tout au long de la période.

\section{TABLEAU IV}

Contributions générales scolaires

Province de Québec, 1867-68, 1887-88, 1907-08, 1927-28

\begin{tabular}{|c|c|c|c|}
\hline Année & Taxes & $\begin{array}{c}\text { Rétributions } \\
\text { mensuelles }\end{array}$ & $\begin{array}{c}\text { Contributions des } \\
\text { institutions } \\
\text { indépendantes }\end{array}$ \\
\hline $1867-1868$ & $25,9 \%$ & $34,5 \%$ & $39,6 \%$ \\
\hline $1887-1888$ & $43,3 \%$ & $6,9 \%$ & $49,8 \%$ \\
\hline $1907-1908$ & $58,8 \%$ & $5,5 \%$ & $35,7 \%$ \\
\hline $1927-1928$ & $62,0 \%$ & $2,1 \%$ & $35,9 \%$ \\
\hline
\end{tabular}

Source: R.S.I.P., $1908-09$ et 1927-28

Il s'avère donc qu'après le début $\mathrm{du} \mathrm{XX}^{\mathrm{e}}$ siècle, les investissements publics pour la construction et le fonctionnement des écoles libèrent la congrégation de l'obligation de fonder un pensionnat pour assurer le service de l'enseignement public. La congrégation des Sœurs de Sainte-Anne peut dorénavant utiliser ses ressources pour agrandir, consolider et transformer ses institutions déjà fondées, assurant ainsi le développement de l'enseignement post-primaire des filles. À titre d'illustration, on trouve au tableau $\mathrm{V}$ l'augmentation de la valeur des propriétés de la congrégation au début du $\mathrm{XX}^{\mathrm{e}}$ siècle. À l'intérieur d'une période de sept ans, cinq des sept établissements appartenant aux religieuses augmentent de valeur. 


\section{TABLEAU $V$}

Valeur des propriétés foncières appartenant

à la congrégation des S.S.A., Province de Québec, 1907, 1913

\begin{tabular}{|c|c|c|c|}
\hline $\begin{array}{c}\text { Année de } \\
\text { fondation }\end{array}$ & $\begin{array}{c}\text { Nom de } \\
\text { l'établissement }\end{array}$ & $\begin{array}{c}\text { Valeur en } \\
1907(\$)\end{array}$ & $\begin{array}{c}\text { Valeur en } \\
1913(\$)\end{array}$ \\
\hline 1853 & St-Jacques & 46200 & 101000 \\
\hline 1857 & St-Cyprien & 18300 & 22000 \\
\hline 1859 & Rigaud & 10000 & 22000 \\
\hline 1861 & Lachine & 80000 & 80000 \\
\hline 1865 & Rawdon & 22000 & 22000 \\
\hline 1874 & St-Cuthbert & 18000 & 25000 \\
\hline 1888 & Ste-Cunégonde & $?$ & $?$ \\
\hline 1898 & L'Ange-Gardien & 78000 & 89000 \\
\hline
\end{tabular}

Source: R.S.I.P., 1907-08, 1913-14

Avant le début du $\mathrm{XX}^{\mathrm{e}}$ siècle, les religieuses avaient trouvé dans la gérance de pensionnats un moyen de financement pour les établissements d'enseignement; à partir de 1902, elles ouvriront essentiellement des écoles et aucun nouveau pensionnat. Au contraire, elles fermeront les portes de deux pensionnats pour les transformer en écoles. À ce stade, il est certes intéressant d'observer que les Sœurs de Sainte-Anne ne possèdent en propre que huit maisons entre 1902 et 1950. Mais, cette constatation doit être accompagnée de l'étude des revenus de ces pensionnats.

\section{Les revenus des pensionnats}

L'étude du budget de fonctionnement des pensionnats nécessite l'établissement d'un choix dans la masse des données disponibles; il serait trop long d'examiner les finances d'une vingtaine de pensionnats. Nous avons donc procédé par sondage: deux décennies de bilans financiers pour quelques 
pensionnats représentatifs de l'ensemble des établissements, petits et gros pensionnats, maisons situées en milieu rural et en milieu urbain ${ }^{43}$.

Les sources de revenus d'un pensionnat sont diversifiées; il est toutefois possible de les regrouper en trois catégories afin de mieux analyser leur provenance. La première catégorie englobe les recettes provenant des coûts de la pension, de l'enseignement régulier, celui de la musique, du dessin, de l'art culinaire et de la dactylographie, ainsi que le matériel scolaire, les fournitures d'ouvrages et les articles d'uniformes. Ces sources ont été

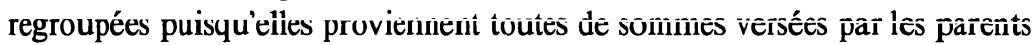
d'élèves inscrites au pensionnat.

La deuxième catégorie regroupe les sources provenant de fonds publics. On en compte deux: la subvention annuelle accordée à toutes les maisons d'enseignement supérieur par le Département de l'instruction publique, et les montants alloués par la Commission scolaire défrayant les salaires des religieuses enseignantes à l'école paroissiale.

Toute autre source de financement pour le pensionnat fait partie de la troisième catégorie: travail des religieuses en dehors de l'enseignement et rémunéré par la paroisse ou la fabrique, surplus de marchandises provenant des terrains et de la basse-cour, intérêts, toute activité du pensionnat source de profits: séances, bazars et finalement quelques dons. Dans cette catégorie, chacune des sources constitue une proportion très faible par rapport à l'ensemble des recettes, c'est pourquoi elles sont traitées globalement et non séparément.

Le tableau VI présente le montant des recettes et le pourcentage du revenu total de chaque catégorie pour divers pensionnats. Ces données sont hétérogènes mais donnent une idée des montants souvent dérisoires que reçoivent les pensionnats.

À l'examen de ces sources, on constate que la première catégorie (pension et enseignement) constitue une large part de la somme totale des revenus de l'institution. Le pourcentage de cette catégorie varie entre chaque maison, mais il demeure généralement plus élevé que celui des autres catégories. À l'intérieur de cette première catégorie, les revenus de la pension, de l'enseignement régulier et celui de la musique - les trois rubriques nous sont données ensemble dans les états financiers - varient entre $82 \%$ et $100 \%{ }^{44}$. Le reste des revenus provient du matériel scolaire, de la fourniture d'ouvrage et des costumes, à l'exception du couvent de Lachine qui possède une source de revenus supplémentaires: les cours privés.

43 Il s'agit des couvents de Vaudreuil (1875-1885 et 1935-1945), St-Rémi (1905-1915 et 1940-1950), Rawdon (1865-1875 et 1905-1915), St-Henri (1895-1905 et 1925-1935) et Lachine (1885-1895, 1915-1925 et 1945-1955), ASSA.

44 ASSA, États financiers des pensionnats. Voir tableau VI. 
TABLEAU VI

Revenus par catégorie de certains des pensionnats de la congrégation des Sœurs de Ste-Anne, Province de Québec

\begin{tabular}{|c|c|c|c|c|c|c|c|c|}
\hline \multirow{2}{*}{$\begin{array}{l}\text { Nom de } \\
\text { l'établissement }\end{array}$} & \multirow{2}{*}{ Année } & \multicolumn{2}{|c|}{$\begin{array}{l}\text { Cat. I - Pension et } \\
\text { enseignement }\end{array}$} & \multicolumn{2}{|c|}{ Cat. II - Fonds publics } & \multicolumn{2}{|c|}{ Cat. III - Divers } & \multirow{2}{*}{$\begin{array}{c}\text { Total } \\
\text { des } \\
\text { revenus } \\
(\$)\end{array}$} \\
\hline & & Revenu (\$) & $\begin{array}{l}\% \text { du revenu } \\
\text { total }\end{array}$ & Revenu (\$) & $\begin{array}{l}\% \text { du revenu } \\
\text { total }\end{array}$ & Revenu (\$) & $\begin{array}{l}\% \text { du revenu } \\
\text { total }\end{array}$ & \\
\hline Rawdon & $1872-73$ & 128,97 & $35,3 \%$ & 133,00 & $36,3 \%$ & 103,95 & $28,4 \%$ & 365,92 \\
\hline Vaudreuil & $1882-83$ & 500,25 & $48,7 \%$ & 221,40 & $21,6 \%$ & 304,71 & $29,7 \%$ & 1026,36 \\
\hline Lachine & $1892-93$ & 13958,89 & $91,5 \%$ & 133,00 & $0,9 \%$ & 1159,64 & $7,6 \%$ & 15251,53 \\
\hline St-Henri & $1902-03$ & 8390,37 & $69,0 \%$ & 1933,00 & $16,0 \%$ & 1822,13 & $15,0 \%$ & 12145,50 \\
\hline St-Rémi & $1912-13$ & 6502,76 & $73,8 \%$ & 464,80 & $5,2 \%$ & 1847,92 & $21,0 \%$ & 8815,48 \\
\hline Rawdon & $1912-13$ & 8766,51 & $77,4 \%$ & 240,30 & $2,1 \%$ & 2318,59 & $20,5 \%$ & 11325,40 \\
\hline Lachine & $1922-23$ & 37780,43 & $88,7 \%$ & 95,00 & $0,2 \%$ & 4720,30 & $11,1 \%$ & 42595,73 \\
\hline St-Henri & $1932-33$ & 14578,43 & $66,9 \%$ & 60,00 & $0,3 \%$ & 7134,73 & $32,8 \%$ & 21773,16 \\
\hline Vaudreuil & $1942-43$ & 9878,73 & $49,4 \%$ & 2849,00 & $14,3 \%$ & 7267,96 & $36,3 \%$ & 19995,69 \\
\hline St-Rémi & $1942-43$ & 13600,40 & $46,8 \%$ & 2415,00 & $8,3 \%$ & 13023,84 & $44,9 \%$ & 29039,24 \\
\hline
\end{tabular}

Source: ASSA, États financiers des pensionnats des S.S.A. 
Les deux autres catégories, soit les fonds publics et les diverses sources de revenus, se divisent l'autre part des recettes, mais la catégorie divers est plus considérable que celle des fonds publics. Cette dernière catégorie comprend deux sources de revenus, les subventions accordées par le Département de l'instruction publique et les salaires versés aux religieuses enseignant à l'école paroissiale. À l'intérieur de cette catégorie, ce sont les salaires des religieuses qui constituent la plus grosse part: $75 \%$ et plus. Les subventions du Département de l'instruction publique viennent compléter cette catégorie. Sur l'ensemble des recettes, ces subventions sont très faibles, elles demeurent généralement sous le $1 \%$ du revenu total du pensionnat.

Pour survivre, les pensionnats ne devaient donc pas se fier aux subventions du gouvernement. De leur côté, les salaires des religieuses enseignant à l'école paroissiale rapportaient peu au couvent, comparé aux fonds nécessaires à l'entretien et à la survie du pensionnat. À défaut de fonds publics suffisants, les religieuses ont dû trouver d'autres sources de revenus afin d'augmenter les recettes annuelles. Nous retrouvons ces sources dans la catégorie divers: confection de cierges et d'hosties pour la paroisse, entretien de la sacristie, activités du pensionnat à base de profits, et surplus de marchandises provenant des cultures et de la basse-cour.

Cet examen sommaire de l'état des revenus des couvents des Sœurs de Sainte-Anne au Québec amène à constater que la survie du pensionnat et de l'externat est due en grande partie aux sources de revenus de la première catégorie, soit l'argent versé par les parents des élèves du pensionnat pour la pension et l'enseignement donnés à leur fille. Ces recettes auront permis de financer le pensionnat et d'entretenir la communauté locale qui enseignait au pensionnat et à l'externat.

Dans un livre récent, Marie-Paule Malouin examine une institution de filles à la fin du XIX $\mathrm{X}^{\mathrm{e}}$ siècle abritant un pensionnat et une école paroissiale. Un chapitre intitulé Dynamique financière examine les revenus de l'établissement et termine en affirmant que "l'école privée «subventionne» l'école publique en cette fin du XIXe siècle ${ }^{45}$. L'examen du chapitre des revenus des pensionnats des Sœurs de Sainte-Anne au Québec nous permet donc de confirmer la conclusion de Marie-Paule Malouin pour notre période, soit de 1850 à 1950 , mais également d'étendre cette observation à l'ensemble d'une congrégation.

45 Marie-Paule Malouin, Ma sœur, à quelle école allez-vous? Deux écoles de filles à la fin du XIX'i siècle, Montréal, Fides, 1985, p. 67. 


\section{Le budget de fonctionnement des institutions}

L'examen des deux principaux chapitres des états financiers, soit les revenus et les dépenses, nous conduit à de nouvelles interrogations; comment fonctionne le budget d'un pensionnat? Quelles sont les dépenses d'une telle institution? Et ces sommes sont-elles suffisantes à son entretien et à la survie des personnes qui y sont attachées?

Au chapitre des dépenses, il y a d'une part les paiements de dettes, soit les montants dus pour annuités ou indemnités et les intérêts sur emprunts contractés pour la plupart à la Maison mère; d'autre part les frais qu'occasionne l'entretien du couvent incluant les couventines et les religieuses, ce que nous désignerons ici comme les frais généraux. Cela comprend le comestible, le chauffage, l'éclairage, la lingerie et vestiaire, le matériel scolaire, les terrains et basse-cour, les cierges, hosties, voyages, ports de lettres, domestiques, ouvriers, vins, liqueurs, remèdes, médecins, et finalement les extras: réparations, achats, assurances, etc.

Le tableau VII présente, pour les mêmes couvents et les mêmes années que dans la partie précédente, le total des recettes, le total des dépenses, l'excédent des recettes sur les dépenses ou vice-versa, et le pourcentage des dépenses par rapport aux recettes, c'est-à-dire le pourcentage des recettes dépensées au cours de l'année.

Même s'il demeure incomplet pour le moment, cet examen de l'état des revenus et dépenses nous permet d'avancer quelques observations. Premièrement, tous les budgets sont excédentaires, on trouve toujours un surplus des recettes sur les dépenses. Cet excédent de recettes varie de 0,6\% à 14,6\% du total des revenus. Il est parfois très mince, comme au couvent de Rawdon en 1912-13 (0,6\%) ou celui de Lachine en 1892-93 (0,8\%). Les dépenses sont alors presque égales aux recettes. Cette balance de caisse, soit l'excédent, est par la suite comptabilisée comme un revenu dans l'état financier de l'année suivante.

On constate en deuxième lieu que la part des frais généraux dans le montant global des dépenses varie entre $76,8 \%$ et $97,5 \%{ }^{46}$. Le reste des dépenses va au remboursement des intérêts, de vieilles dettes et des emprunts. Ainsi à chaque année les pensionnats dépensent en moyenne $13 \%$ de leur budget annuel pour le paiement de vieilles dettes, et la plus grande part, soit $87 \%$ est utilisée pour les besoins du pensionnat.

46 Ces données proviennent des états financiers des couvents déjà présentés dans les deux tableaux précédents et ce, pour les mêmes années. Nous n'avons pas vu l'utilité de présenter ici un tableau supplémentaire sur les dépenses des pensionnats: ces quelques résultats résument toutes les données. 
TABLEAU VII

Recettes par catégorie de certains des pensionnats de la congrégation des Sours de Ste-Anne, Province de Québec

\begin{tabular}{|c|c|c|c|c|c|}
\hline $\begin{array}{c}\text { Nom de } \\
\text { l'établissement }\end{array}$ & Année & Total des recettes $(\$)$ & Total des dépenses (\$) & $\begin{array}{l}\text { Excédent des recettes } \\
\text { sur les dépenses }(\$)\end{array}$ & $\begin{array}{c}\text { Pourcentage } \\
\text { des dépenses par } \\
\text { rapport aux recettes }\end{array}$ \\
\hline Rawdon & $1872-73$ & 365,92 & 348,59 & 17,33 & $95,3 \%$ \\
\hline Vaudreuil & $1882-83$ & 1026,36 & 946,43 & 79,93 & $92,2 \%$ \\
\hline Lachine & $1892-93$ & 15251,53 & 15126,43 & 125,10 & $99,2 \%$ \\
\hline St-Henri & $1902-03$ & 12145,50 & 11686,41 & 459,09 & $96,2 \%$ \\
\hline St-Rémi & $1912-13$ & 8815,48 & 7789,15 & 1026,33 & $88,4 \%$ \\
\hline Rawdon & $1912-13$ & 11325,40 & 11262,43 & 62,97 & $99,4 \%$ \\
\hline Lachine & $1922-23$ & 42595,73 & 41763,53 & 832,20 & $98,0 \%$ \\
\hline St-Henri & $1932-33$ & 21773,16 & 20542,92 & 1230,24 & $94,3 \%$ \\
\hline Vaudreuil & $1942-43$ & 19995,69 & 17076,80 & 2918,89 & $85,4 \%$ \\
\hline St-Rémi & $1942-43$ & 29039,24 & 27982,16 & 1057,08 & $96,4 \%$ \\
\hline
\end{tabular}

Source: ASSA, États financiers des pensionnats des S.S.A. 
En dernier lieu, nous présentons les résultats d'un exercice de calcul sur l'évaluation des dépenses annuelles par personne attachée au pensionnat. Il a été possible d'arriver à ce montant en divisant le total des frais généraux, soit le total des dépenses moins les dettes, par le nombre de personnes reliées à l'établissement, incluant les élèves de l'internat et les religieuses enseignant au pensionnat et à l'externat (tableau VIII). Les données provenant des états financiers concernent les mêmes établissements et les mêmes années que les trois tableaux précédents, tandis que le nombre de personnes, soit les élèves et les religieuses, provient des livres de comptes de chaque maison. Les résultats de ce calcul sont présentés dans la dernière colonne du tableau. Ces montants sont difficiles à interpréter, mais leur intérêt et leur validité augmenteront en les comparant à des données analogues.

\section{TABLEAU VIII}

Dépenses annuelles par personne, Institutions des S.S.A., Province de Québec

\begin{tabular}{|cc|c|c|c|c|c|}
\hline $\begin{array}{cc}\text { Nom de } \\
\text { l'établis- } \\
\text { sement }\end{array}$ & Année & $\begin{array}{c}\text { Frais généraux } \\
\text { (dépenses moins } \\
\text { dettes) }\end{array}$ & $\begin{array}{c}\text { Nombre de } \\
\text { religieuses } \\
\text { (int. et ext.) }\end{array}$ & $\begin{array}{c}\text { Nombre } \\
\text { d'élèves } \\
\text { (internat) }\end{array}$ & $\begin{array}{c}\text { Nombre } \\
\text { total de } \\
\text { personnes }\end{array}$ & $\begin{array}{c}\text { Dépenses } \\
\text { annuelles } \\
\text { par personne } \\
\text { (dollars) }\end{array}$ \\
\hline Rawdon & $1872-73$ & 267,59 & 3 & 10 & 13 & 20,58 \\
\hline Vaudreuil & $1882-83$ & 782,84 & 6 & 48 & 54 & 14,50 \\
\hline Lachine & $1892-93$ & $3386,36^{1}$ & 29 & 201 & 230 & $14,72^{2}$ \\
\hline St-Henri & $1902-03$ & 9816,41 & 28 & 110 & 138 & 71,13 \\
\hline St-Rémi & $1912-13$ & 6709,15 & 16 & 122 & 138 & 48,62 \\
\hline Rawdon & $1912-13$ & 10224,22 & 14 & 125 & 139 & 73,56 \\
\hline Lachine & $1922-23$ & $7400,53^{1}$ & 41 & 165 & 206 & $35,92^{2}$ \\
\hline St-Henri & $1932-33$ & 19642,92 & 30 & 72 & 102 & 192,58 \\
\hline Vaudreuil & $1942-43$ & 13917,80 & 18 & 70 & 88 & 158,16 \\
\hline St-Rémi & $1942-43$ & 27292,16 & 23 & 106 & 129 & 211,57 \\
\hline
\end{tabular}

Sources: ASSA, États financiers des pensionnats des S.S.A.

1. Ce chiffre est sous-évalué car une grande partie des frais généraux est comprise dans le montant envoyé à la Maison mère, constitué ailleurs uniquement des dettes et des emprunts.

2. Ce chiffre est inexact à cause du montant des frais généraux. 
Un problème majeur apparaît à ce stade: à notre connaissance, aucune étude ne présente de renseignements de même type. Seul l'ouvrage d'Arthur St-Pierre sur les œuvres de charité ${ }^{47}$ propose quelques éléments comparables à nos chiffres pour le début des années 1930. Par exemple, chez les Sœurs Grises de Montréal, on trouve une dépense annuelle moyenne de $261 \$$ par lit pour les treize institutions qu'elles possèdent. Chez les Sœurs du BonPasteur de Montréal, l'hospitalisation annuelle d'un enfant coûte entre $206 \$$ et $238 \$$ par lit. Du côté des Frères de St-Gabriel, les dépenses annuelles à l'orphelinat sont de $140 \$$ par lit, et de $268 \$$ au patronage. Chez les Clercs de St-Viateur, les frais d'administration couvrant la pension, l'instruction régulière et la formation professionnelle dans un institut pour sourds-muets sont de $250 \$$. Pour une maternité-crèche, les chiffres varient: la dépense moyenne annuelle est de 200 \$ chez les Sœurs du Bon-Pasteur de Québec; de $333 \$$ chez les Sœurs Grises de Montréal; de $400 \$$ chez les Sœurs de la Miséricorde; et de $730 \$$ à $1500 \$$ ou plus dans des établissements sembables en Ontario et aux États-Unis ${ }^{48}$. Rappelons que tous ces montants ont été calculés pour l'année 1930; ils sont similaires aux dépenses annuelles de nos pensionnats pour la même période. On doit préciser que nos calculs couvrent une période de douze mois, et qu'en réalité, les élèves ne demeurent au pensionnat que dix mois par année.

En examinant ces chiffres dérisoires, on ne peut douter du travail qu'ont accompli les religieuses dans leurs institutions. Dans son étude, Arthur St-Pierre avance cette explication pour les congrégations religieuses de charité: "C'est le travail gratuit ou quasi gratuit du personnel religieux surtout qui permet à ces [...] maisons de fonctionner aussi économiquement ${ }^{49}$. De toute évidence, cette explication semble valable également pour les institutions éducatives.

L'évolution globale des moyennes annuelles observées entre 1872 et 1942 réfère vraisemblablement à l'augmentation globale du coût de la vie et permet de croire qu'un même fonctionnement «économique» a prévalu, durant toute la période, pour la majorité des pensionnats.

\section{8. Émergence d'un modèle de fonctionnement}

Quel modèle peut-on dégager des données que nous venons d'exposer? Il faut d'abord faire ressortir la distinction entre le $\mathrm{XIX}^{\mathrm{e}}$ siècle et le $\mathrm{XX}^{\mathrm{e}}$ siècle, due principalement à l'augmentation des investissements éducatifs de

47 Arthur St-Pierre, L'œuvre des congrégations religieuses de charité dans la Province de Québec (en 1930), Montréal, Éditions de la Bibliothèque canadienne Enrg., 1930, 248 p.

48 Ibid., p. 107, 154, 167, 169, 194.

49 Ibid, p. 167. 
l'État. Au XIXe siècle, pour pouvoir se maintenir dans les écoles où on les réclame, les religieuses doivent ouvrir un pensionnat. La plupart du temps, elles ne sont que locataires des maisons qu'elles fondent: ce sont les fabriques paroissiales qui fournissent bâtisses et terrains. Pour attirer la clientèle payante qui assurera les revenus indispensables à la survie, elles doivent offrir un produit de meilleure qualité (une scolarité plus longue) au pensionnat qu'à l'externat. Toutes les maisons fondées par les S.S.A. au XIXe siècle sont des pensionnats/externats. Elles ne fondent aucun nouveau pensionnat entre 1900 et 1950.

Avec le début du XXe siècle, l'État québécois, par le biais des autorités scolaires, accepte d'investir les sommes suffisantes pour faire fonctionner des écoles, surtout dans les villes. Effectivement, la congrégation des Sœurs de Sainte-Anne ouvre 45 écoles en 50 ans. Mais en même temps, elle continue de recevoir des pensionnaires dans ses vingt pensionnats. La plupart, on l'a vu, sont jumelés à des écoles. Mais les salaires des religieuses enseignant dans les classes de l'école publique, même s'ils sont plus élevés qu'au siècle précédent, génèrent des revenus minimes. Le pensionnat doit continuer de s'autofinancer à même les revenus des pensions. Et pour maintenir la clientèle, les religieuses se démarquent du secteur public, d'abord en offrant une scolarité plus longue, puis, lorsque l'école publique s'allonge, en proposant des programmes différents: cours Lettres-Sciences, cours d'école normale, cours supérieur d'enseignement ménager, cours classique. L'apparition de ces nouveaux cours se produisant dans plusieurs congrégations, on peut affirmer qu'il s'agit là d'un modèle applicable à plus d'une congrégation.

Tous les pensionnats que nous avons examinés vivent presque exclusivement des pensions des élèves et des "extras" que procurent les bazars et autres activités jusqu'en 1950. De plus, au chapitre du fonctionnement, nous n'avons noté aucun déficit, ce qui dénote une gestion très serrée.

Les investissements immobiliers (construction, réparation, agrandissement) sont gérés de la maison mère, et chaque pensionnat peut y emprunter les sommes nécessaires à ses opérations, mais doit, par la suite, rembourser la maison mère à même le "surplus" de son budget annuel. La maison mère fournit les sommes requises en hypothéquant les édifices déjà construits. L'examen du passif des opérations immobilières démontre une extrême prudence, chaque dette ou hypothèque étant patiemment résorbée, année par année.

Il est très net aussi que le nombre de religieuses sans salaire est considérable dans l'ensemble de la congrégation. En effet, une école peut accueillir trois ou quatre religieuses alors qu'un pensionnat, même modeste, en requiert souvent plus de quinze. La véritable «économie» de ce modèle de fonctionnement financier est assurée par la gratuité du travail des 
religieuses. C'est ce qui explique d'ailleurs la différence entre le coût d'une école normale de filles et une de garçons notée en introduction.

En fait, il faudra attendre la période qui suit la seconde guerre mondiale pour que l'État collabore à la construction de nouvelles maisons (l'institut familial de St-Jacques par exemple), accorde des subventions plus généreuses, augmente le salaire des enseignantes à l'école publique, permettant ainsi à la congrégation de maintenir ses pensionnats jusqu'à la réforme Parent sans augmenter de façon substantielle le coût de la pension et des services éducatifs.

Lorsque les collèges classiques féminins présentent leur mémoire à la Commission royale d'enquête sur les problèmes constitutionnels en 1954, les responsables n'ont pas de mal à démontrer que les collèges classiques féminins sont des institutions déficitaires et que la justice la plus élémentaire requiert qu'ils soient subventionnés comme les collèges masculins ${ }^{50}$. De toute évidence, elles auraient pu conduire la même analyse pour la plupart de leurs pensionnats. Pourtant, les Sœurs de Sainte-Anne réussissent, en 1956, à construire au coût de 4478091 \$, et à faire fonctionner une entreprise colossale, l'Institut Cardinal-Léger (futur cegep de Rosemont), où coexistent une école normale, un scolasticat, un cours classique du premier degré, une école d'application et un jardin d'enfants ${ }^{51}$.

La congrégation des Sœurs de Sainte-Anne est-elle riche? De toute évidence, il est impossible de répondre à cette question qui exigerait, au moins, un éventail de comparaisons. Tout au plus peut-on faire les observations suivantes. En 1950, la congrégation possède au Québec quelques immeubles, une dizaine, dont la valeur foncière servira à cautionner l'hypothèque qui va permettre la construction de l'Institut Cardinal-Léger. Les deux-tiers de ses 2095 religieuses professes sont réparties dans les institutions québécoises. De ce nombre, on peut proposer les évaluations suivantes: 487 religieuses enseignant dans les écoles publiques ${ }^{52}$ et leurs salaires annuels peuvent atteindre la somme globale de $487000 \$ \$^{53}$; près de 700 religieuses travaillent sans aucun salaire dans les divers pensionnats et dans les postes de gestion interne de la congrégation $^{54}$; quelque 180 religieuses âgées (70 ans) et retraitées reçoivent la pension de vieillesse du

50 La signification et les besoins de l'enseignement classique pour jeunes filles, op. cit.

51 Louise Roy, s.s.a., Institut et école normale Cardinal-Léger, Montréal, texte miméographié, 1974, p. 32 . Le 12 novembre 1969, la congrégation vendait cette institution au gouvernement provincial pour la somme de 3636400 \$. Ibid., p. 163. Cet «épisode» mériterait un traitement à part qui déborde les cadres de cet article.

52 Le Canada ecclésiastique, 1950, p. 914-922.

53 D'après le tableau du Rapport du Surintendant de l'Instruction publique, 1950-51, p. 56.

54 Le Canada ecclésiastique, op. cit. 
gouvernement fédéral pour un montant global annuel de $86400 \$ 55$; les revenus générés par les pensionnats servent exclusivement à assurer les services requis par l'internat. En somme, la totalité de leurs revenus sert à défrayer les dépenses indispensables à leur survie matérielle et les surplus sont investis systématiquement dans la création et le fonctionnement d'institutions éducatives. Si au XIX ${ }^{e}$ siècle, le pensionnat privé servait à financer l'école publique, après 1950, c'est un mouvement contraire qui pourra s'établir et on peut penser que l'entreprise de Cardinal-Léger ne sera possible qu'à partir du moment où les salaires des enseignantes se mettront à augmenter. Mais l'évolution de la congrégation connaîtra, avec la baisse des entrées, le départ de religieuses professes et le vieillissement des sujets, une conjoncture nouvelle qui brisera ce fragile équilibre financier.

On obtient une autre approximation en examinant la situation actuelle de la congrégation. En effet, la démocratisation de l'enseignement secondaire, après 1954 et surtout 1965, allait donner le signal de la fermeture de ses maisons d'éducation. Aujourd'hui, la congrégation en maintient quelquesunes dans le réseau privé: quatre au niveau secondaire et une au niveau primaire où, en 1984, œuvraient 193 religieuses; par ailleurs, la congrégation comptait, à la même date, 121 enseignantes au secteur public dont les salaires, on le conçoit, étaient indispensables au soutien de la congrégation ${ }^{56}$.

Le modèle de financement de la congrégation des Sœurs de Sainte-Anne peut-il s'appliquer aux autres congrégations? Il serait prématuré de l'affirmer mais tout indique que les congrégations fondées après 1840 qui ont eu un développement institutionnel semblable à celui des Sœurs de Sainte-Anne, réseau primitif de pensionnats suivi, au $\mathrm{XX}^{\mathrm{e}}$ siècle, d'un réseau important d'écoles publiques, ont connu un modèle de financement semblable.

55 Matricule des Sœurs de Ste-Anne pour le nombre de retraitées et Statistiques Canada. Sécurité sociale. vol. 6: Programmes nationaux, Allocations et services sociaux, Ottawa, p. 14 (1982) pour le montant annuel des pensions en 1950: 480\$.

56 AREQ, Association des religieuses enseignantes du Québec, vol. 23, no 4 (mai 1984), p. 23. 